\title{
Respuesta del duraznero a diferentes patrones de aplicación del agua
}

\author{
Pablo Morales ${ }^{1}$, Mario García-Petillo ${ }^{1}$, Raquel Hayashi ${ }^{1}$ \& Lucía Puppo ${ }^{1}$
}

\begin{abstract}
RESUMEN
Se realizó un experimento durante tres años (2004/05 a 2006/07) sobre un monte de duraznero recién implantado en el sur de Uruguay. Se aplicaron cinco tratamientos de riego: secano (T1), goteros de 1,6 $\mathrm{L} \mathrm{h}^{-1}$ a $0,4 \mathrm{~m}$ (T2), goteros de $4 \mathrm{~L} \mathrm{~h}^{-1}$ a $1 \mathrm{~m}$ (T3), microjets de $21 \mathrm{~L} \mathrm{~h}^{-1}$ a $5 \mathrm{~m}$ (T4) y goteros de $2 \mathrm{~L} \mathrm{~h}^{-1}$ a $1 \mathrm{~m}$, dos líneas por fila (T5), en un diseño experimental en cuadro latino. Las parcelas eran de siete plantas en un marco de plantación de 2,5 x 4,5 m. En todos los tratamientos con riego se aplicó la misma dosis de agua, de forma de cubrir el $100 \%$ de la ETc. El porcentaje de volumen de suelo mojado fue de 16, 18, 22 y 44\% para los tratamientos T2, T3, T4 y T5 respectivamente. En las variables peso de poda, potencial hídrico foliar y xilemático, conductancia estomática, crecimiento de frutos, rendimiento y tamaño promedio de los frutos, los tratamientos que recibieron riego no se diferenciaron estadísticamente entre sí, pero todos presentaron valores significativamente superiores al secano $(p<0,05)$. No se evidenció ninguna respuesta entre los tratamientos regados al porcentaje de suelo mojado, para todas las variables analizadas. Se plantea la hipótesis que los efectos de estos tratamientos, en un cultivo perenne, serían acumulativos y se podrían expresar en años sucesivos.
\end{abstract}

Palabras-clave: patrones de mojado, Prunus persica, riego localizado, volumen de suelo mojado

\section{Peach trees response to different water application patterns}

\begin{abstract}
An experiment was carried out for three years (2004/05 to 2006/07) on a just implanted peach grove, in the south of Uruguay. Five irrigation treatments were applied: without irrigation (T1), drippers of $1.6 \mathrm{~L} \mathrm{~h}^{-1}$ at $0.4 \mathrm{~m}$ (T2), drippers of $4 \mathrm{~L} \mathrm{~h}^{-1}$ at $1 \mathrm{~m}$ (T3), microjets of $21 \mathrm{~L} \mathrm{~h}^{-1}$ at $5 \mathrm{~m}$ (T4) and drippers of $2 \mathrm{~L} \mathrm{~h}^{-1}$ at $1 \mathrm{~m}$, two lines by row (T5), following a Latin square experimental design. The experimental plots had seven trees in a plantation frame of $2.5 \times 4.5 \mathrm{~m}$. In all treatments with irrigation, the same water dose was applied, in order to fulfill $100 \%$ of the ETc. The percentage of wetted soil volume was 16, 18, 22 and 44\% for treatments T2, T3, T4 and T5 respectively. Regarding variables, prune weight, foliar and xilematic water potential, stomata conductance, fruit growth, yield and average size of the fruits, the treatments with irrigation were not statistically different, but all of them were significantly different from the treatment without irrigation $(p<0.05)$. No response to the percentage of wet soil among the irrigated treatments was detected, for all the analyzed variables. The hypothesis that the effects of these treatments, upon a perennial crop would be cumulative, and would possibly show up in the following years, is considered.
\end{abstract}

Key words: wetting patterns, Prunus persica, microirrigation, soil wetted volume 


\section{INTRODUCCIÓN}

En Uruguay la fruticultura ocupa una superficie de 8575 ha de las cuales $50 \%$ corresponde a manzanos (Malus domestica Borkh), el 30\% corresponde a duraznero (Prunus persica L. Batch) y el $14 \%$ a peral (Pyrus communis L.) (MGAP, 2006). La producción de duraznos está destinada principalmente al mercado interno con valores promedios de 15800 t. En las condiciones climáticas de Uruguay si bien se registran precipitaciones con valores anuales promedios de $1200 \mathrm{~mm}$, ocurren períodos de déficit hídricos durante la estación de crecimiento que afectan la producción y la calidad de la fruta cosechada. Por consiguiente, cada vez es más frecuente el uso de riego suplementario en el cultivo de duraznero, con una superficie bajo riego de $51 \%$ del total plantada (MGAP, 2006).

El principal sistema de riego utilizado en frutales en Uruguay y en el mundo corresponde al riego localizado, el cual utiliza pequeños caudales a baja presión, alta frecuencia y no moja la totalidad del suelo (López et al., 1992). Black \& West (1974) trabajando en manzanos jóvenes obtuvieron una reducción de tan solo el 25\% de la evapotranspiración del cultivo (ETc) al reducir el volumen de suelo mojado a un 25\%. En función de este experimento básico, varios investigadores han realizado trabajos a efectos de determinar el porcentaje de suelo mojado necesario para no afectar los rendimientos en naranjas (Moreshet et al., 1983; Koo \& Smajstrla, 1985) duraznero, almendros y en olivos (Willoughby \& Cockroff, 1974; Fereres, 1981; Gispert, 2003). Según Keller (1978), no ha sido establecido un valor mínimo absoluto para el porcentaje de suelo mojado y tentativamente aconseja para cultivos ampliamente espaciados, porcentajes superiores al 20\% en zonas de alta precipitación y suelos de textura arcillosa, y entre 33 a 55\% en zonas de baja precipitación.

Las investigaciones de repuesta al porcentaje de suelo mojado en frutales de hoja caduca y especialmente en climas húmedos, actualmente son muy escasas. En el Uruguay se realizó un experimento de respuesta al volumen de suelo mojado en naranja "Washington Navel”, obteniéndose un incremento de $6 \%$ en el rendimiento al aumentar el porcentaje de suelo mojado de 20 a 35\% (García-Petillo et al., 2004). En un trabajo posterior, García-Petillo et al. (2005) establecieron que en los suelos estratificados y pesados, típicos de la zona frutícola sur del Uruguay, los patrones de mojado en riego por goteo fueron sustancialmente diferentes a los esperables de acuerdo a la bibliografía. El ancho de la banda mojada fue generalmente menor a lo esperado y en situaciones de riego tal cual lo manejan los productores, muchas veces ni siquiera se solapan los bulbos mojados. Las pérdidas por percolación detectadas fueron importantes, por lo que se está sobreestimando la eficiencia de aplicación del método, y por lo tanto sub-irrigando el cultivo.

En este trabajo se plantea como objetivo evaluar la respuesta vegetativa y productiva de un monte joven de duraznero cv. "Dixiland" al variar el patrón de mojado mediante el uso de diferentes emisores.

Este objetivo se fundamenta en la necesidad de realizar investigación nacional a los efectos de generar la información que permita el correcto diseño y manejo del riego localizado en las condiciones de Uruguay.

\section{MATERIAL Y MÉTODOS}

El ensayo se instaló en un monte de duraznero (Prunus persica L. Batch) en el Centro Regional Sur de la Facultad de Agronomía, $34^{\circ} 37^{\prime} \mathrm{S}, 56^{\circ} 13^{\prime} \mathrm{W}$, a $31 \mathrm{~km}$ al norte de la ciudad de Montevideo.

El monte se implantó en setiembre de 2003. Se utilizó una variedad de estación cv "Dixiland" injertada sobre pie franco de pavía moscatel. El marco de plantación fue de $4,5 \mathrm{~m}$ en la entrefila por $2,5 \mathrm{~m}$ en fila, lo que determina una densidad de plantación de 888 árboles por hectárea. El sistema de conducción fue en vaso moderno con tres líderes.

El suelo del ensayo es un Argiudol Páchico, fino esmectítico térmico (Durán et al., 2005), con la siguiente descripción (Tabla 1):

Tabla 1. Descripción del suelo del ensayo y parámetros hídricos del mismo

\begin{tabular}{|c|c|c|c|c|c|c|}
\hline \multirow{2}{*}{ Horizonte } & \multirow{2}{*}{$\begin{array}{l}\text { Prof. } \\
\text { (cm) }\end{array}$} & \multirow{2}{*}{ Textura } & \multirow{2}{*}{$\begin{array}{c}\text { D. Ap. } \\
\left(\mathrm{g} \mathrm{cm}^{-3}\right)\end{array}$} & CC & PMP & A.D. \\
\hline & & & & \multicolumn{3}{|c|}{$\left(\mathrm{mm} \mathrm{dm}^{-1}\right)$} \\
\hline A & $0-25$ & Fr Lim & 1.12 & 31 & 18 & 13 \\
\hline B & $25-45$ & Fr Arc Lim & 1.36 & 36 & 22 & 14 \\
\hline $\mathrm{BC}$ & $45-75$ & Fr Arc Lim & 1.35 & 40 & 23 & 17 \\
\hline
\end{tabular}

Los tratamientos se comenzaron a aplicar a partir de la segunda temporada de crecimiento (2004-2005), debido a que en el primer año se regaron todos los árboles a los efectos de asegurar la correcta implantación de los mismos.

Se aplicaron cinco tratamientos de riego en un diseño de cuadro latino, con cinco repeticiones. Las parcelas eran de siete árboles contiguos de los cuales se evaluaron los cinco centrales.

Los tratamientos fueron:

T1) Secano

T2) Goteros de 1,6 $\mathrm{L} \mathrm{h}^{-1}$ a $0,4 \mathrm{~m}$

T3) Goteros de $4 \mathrm{~L} \mathrm{~h}^{-1}$ a $1 \mathrm{~m}$

T4) Microjets de $21 \mathrm{~L} \mathrm{~h}^{-1}$ a $5 \mathrm{~m}$

T5) Goteros de $2 \mathrm{~L} \mathrm{~h}^{-1}$ a $1 \mathrm{~m}$, con dos líneas por fila.

El caudal de todos los tratamientos regados era, por lo tanto, $4 \mathrm{~L} \mathrm{~h}^{-1} \mathrm{~m}^{-1}$. A estos cuatro tratamientos se les aplicó la misma dosis de agua, equivalente al $100 \%$ de la $\mathrm{ET}_{\mathrm{c}}$, y la diferencia entre ellos fue el patrón de mojado. $\mathrm{La} \mathrm{ET}_{\mathrm{c}}$ se estimó mediante la metodología de Penman-Monteith (Allen et al., 1998), utilizando los datos climáticos obtenidos de una estación automática Davis modelo Vantage Pro instalada en el sitio del experimento.

Se realizaron muestreos para la determinación de la forma y tamaño de los bulbos mojados en los cuatro tratamientos con riego y también en el T1 (secano) como control. Los mismos se repitieron en dos fechas diferentes, el 12 de noviembre y el 21 de diciembre de 2005, con tiempos de riego de 2 h y 3 h 15 min, respectivamente. 
Para el muestreo se sacaron muestras de suelo a intervalos de profundidad de $0,20 \mathrm{~m}$, utilizando un taladro tipo holandés.

La cuadrícula se conformó por tres transectas perpendiculares a la línea de goteo, una debajo del gotero, otra en el punto medio entre dos goteros y la tercera en el punto medio entre las dos anteriores. En cada una de ellas se marcaron sitios a intervalos de $0,20 \mathrm{~m}$ desde el gotero hacia ambos lados de la línea. Se utilizaron tantos sitios y profundidades como fue necesario para incluir todo el bulbo mojado en cada caso.

Se determinó el contenido de humedad de las muestras mediante el método gravimétrico y con el valor de densidad aparente correspondiente a cada horizonte, se calculó el contenido de agua volumétrico.

Durante las tres temporadas de evaluación (2004-2007) se realizaron determinaciones semanales de la humedad de suelo, mediante una sonda de neutrones marca CPN modelo 503DR HYDROPROBE, en tres parcelas por tratamiento. Cada tubo de acceso estaba situado en la fila, equidistante de dos goteros contiguos.

Las variables de crecimiento vegetativo evaluadas fueron sección de tronco y peso de poda (el crecimiento de brotes y el volumen de copa fue medido pero aquí no se presentan estos resultados). Las determinaciones se realizaron en los cinco árboles de cada parcela (125 árboles en total).

La sección de tronco se estimó a partir de la medición de la circunferencia. Ésta se realizó cada cuatro meses aproximadamente en los períodos invernales y cada dos meses en la estación de crecimiento. Las podas se hicieron cada vez que fue necesario por razones de manejo y conducción del árbol, siendo bastante frecuentes en estos primeros años, ya que se estaba en la etapa de formación de los mismos.

Se midió la conductancia estomática utilizando un porómetro marca DELTA-T modelo AP4 en tres a seis hojas por tratamiento, en tres fechas diferentes y en diferentes horas a lo largo del día.

También se midió el potencial hídrico foliar, utilizando una cámara de presión marca Soilmoisture modelo 30051412, en tres a seis hojas por tratamiento, siguiendo los procedimientos descritos por Scholander et al. (1965). Las mediciones se hicieron antes del amanecer, a media mañana y a máxima demanda atmosférica.

En la temporada 2007 en lugar del potencial hídrico se midió el potencial xilemático, cubriendo las hojas una hora antes de la medición con un film plástico y sobre éste uno de aluminio, siguiendo la metodología de Shackel (2001).

Se realizaron mediciones de crecimiento de frutos en cinco frutos por árbol en dos árboles por parcela, totalizando 50 frutos por tratamiento, durante las temporadas 2005/2006 y 2006/2007. En cada fruto se tomaban, utilizando un calibre digital electrónico, dos medidas de diámetro perpendiculares entre sí las que luego se promediaban.

La primera cosecha de importancia comercial fue la de la temporada 2006-2007. Se evaluó el rendimiento de cada uno de los árboles, el número de frutos de los mismos y en base a estos dos valores se estimó su peso promedio. Se tomaron muestras compuestas de 150 frutos por parcela, los que se calibraron y separaron en tres categorías comerciales: especial (mayores a 180 g), grandes (120 a 180 g) y chicos (menores de $120 \mathrm{~g}$ ).

\section{RESULTADOS Y DISCUSIÓN}

\section{Clima y riego aplicado}

La evapotranspiración del cultivo fue incrementándose en los tres años sucesivos, a medida que los árboles aumentaban su tamaño (Tabla 2).

Tabla 2. Fecha de inicio y fin del riego, riego total aplicado (mm), ET estimada del cultivo $(\mathrm{mm})$ y lluvias totales $(\mathrm{mm})$ en las tres temporadas de evaluación del ensayo

\begin{tabular}{lccc}
\hline & $\mathbf{2 0 0 4 / 2 0 0 5}$ & $\mathbf{2 0 0 5 / 2 0 0 6}$ & $\mathbf{2 0 0 6 / 2 0 0 7}$ \\
Período & $18 / 10-12 / 04$ & $17 / 10-18 / 05$ & $12 / 10-02 / 03$ \\
Riego & 170 & 404 & 210 \\
ETc & 341 & 570 & 702 \\
Lluvia & 924 & 511 & 447 \\
\hline
\end{tabular}

En la primera temporada, a pesar de las altas precipitaciones registradas que superaron ampliamente a la evapotranspiración del cultivo, fue necesario aplicar $170 \mathrm{~mm}$ de riego. Esto se debió a la irregular distribución de las mismas, con lluvia por debajo de la media en diciembre y enero y muy por encima en febrero.

En la temporada 2005/2006 las lluvias fueron inferiores a la media en la primavera (octubre a diciembre) y luego alrededor de la media en verano (enero a marzo). A pesar de esto, fue necesario aplicar más de $400 \mathrm{~mm}$ de riego.

En la última temporada (2006/2007), durante la primavera llovió alrededor de la media, pero casi no se registraron precipitaciones en enero. Debido a problemas operativos con el equipo de riego, la cantidad de agua aplicada no llegó, en algunas ocasiones, a cubrir la demanda del cultivo. A la fecha del 2 de marzo, se deberían de haber regado más de los $210 \mathrm{~mm}$ que se habían aplicado hasta ese momento.

\section{Evolución de la humedad en el suelo}

En la temporada 2004/2005, el contenido de agua en los tres tratamientos regados por goteo (T2, T3, T5) se mantuvo siempre próximo a capacidad de campo (CC). Por el contrario, el tratamiento regado por microjet (T4) tuvo casi exactamente el mismo comportamiento que el secano (T1).

El ensayo se manejó regando simultáneamente los cuatro tratamientos. Para esto, los tiempos y frecuencias de riego se calcularon en función del riego por goteo. Si se hubiera manejado considerando prioritariamente el microjet, se hubiera elegido una menor frecuencia y por lo tanto mayores tiempos de riego. En definitiva, el manejo tal cual se hizo perjudicó el tratamiento con microjet en beneficio de los tratamientos con goteros, especialmente en esta temporada en que los árboles eran muy chicos y por lo tanto las láminas de riego muy reducidas.

En la temporada 2005/2006 el T2 y T5 permanecieron en 
un rango adecuado de humedad, mientras que el T1 y T4, por las mismas causas del año anterior, descendieron por debajo del punto de marchitéz permanente (PMP). En esa temporada también se nota una caída en el contenido de humedad en el T3. Este tratamiento es con goteros de $4 \mathrm{~L} \mathrm{~h}^{-1}$ a $1 \mathrm{~m}$, y el tubo de acceso para la sonda está ubicado entre ambos. Este descenso estaría mostrando que a la profundidad considerada $(0-45 \mathrm{~cm})$, los bulbos no se solapan suficientemente.

En la temporada 2006/2007, el comportamiento es similar al año anterior, con la salvedad que hacia el final de la temporada, debido a los problemas operativos del equipo de riego ya señalados, el contenido de agua en todos los tratamientos regados tuvo una tendencia a descender (Figura 1).

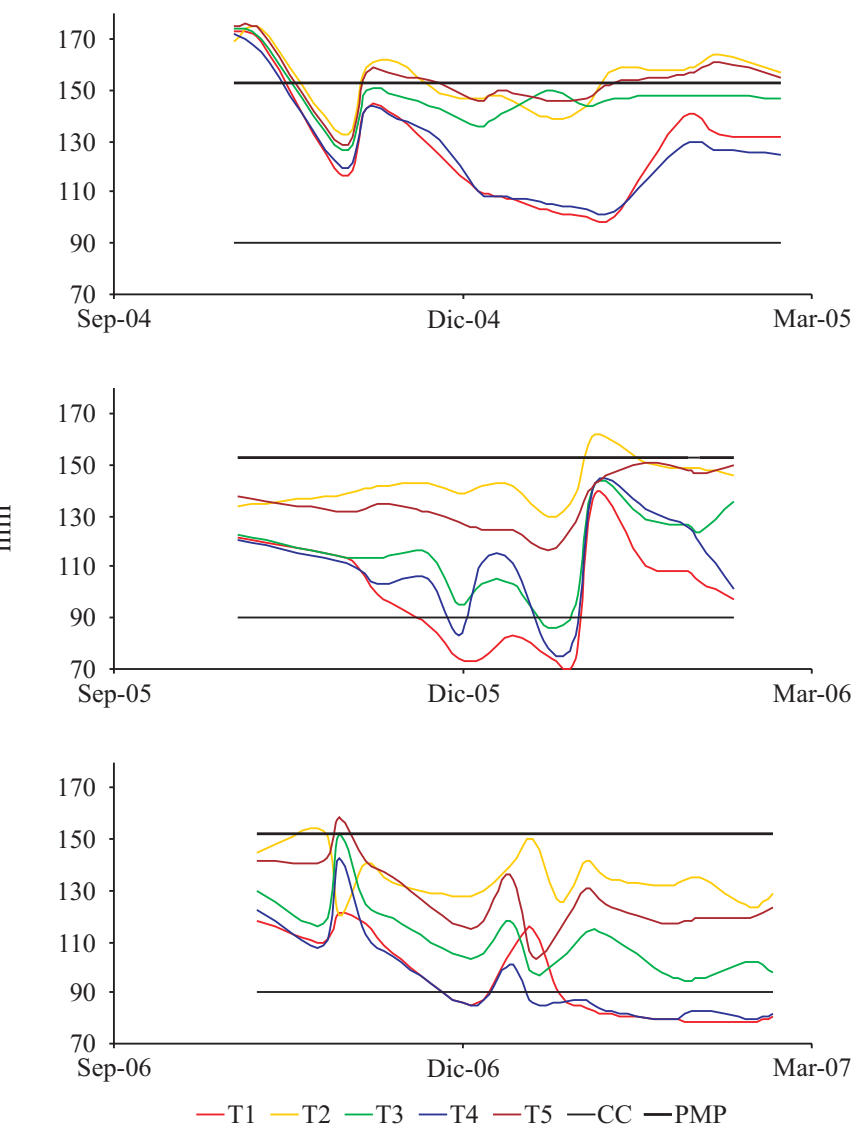

Figura 1. Evolución de la humedad del suelo en los cinco tratamientos, en las temporadas 2004/05, 2005/06 y 2006/07. CC - capacidad de campo, PMP - punto de marchitéz permanente. Todos los valores en mm totales en el perfil de 0 a $45 \mathrm{~cm}$

\section{Forma de los bulbos mojados}

En este trabajo sólo se presentan los resultados correspondientes al muestreo realizado el 21 de diciembre de 2005, con un tiempo de riego de $3 \mathrm{~h} 15$ min (Figura 2). Los valores corresponden a una transecta perpendicular a la fila, bajo el gotero y no se presentan los resultados de las otras transectas.

En la Figura 2, la escala corresponde a contenido de humedad del suelo expresado como porcentaje respecto al agua disponible total del perfil. Un valor de 100 (colores verdes) corresponde a CC y un valor de 0 (colores rojos) corresponde al PMP. Los valores en el eje horizontal son distancias, en cm. El
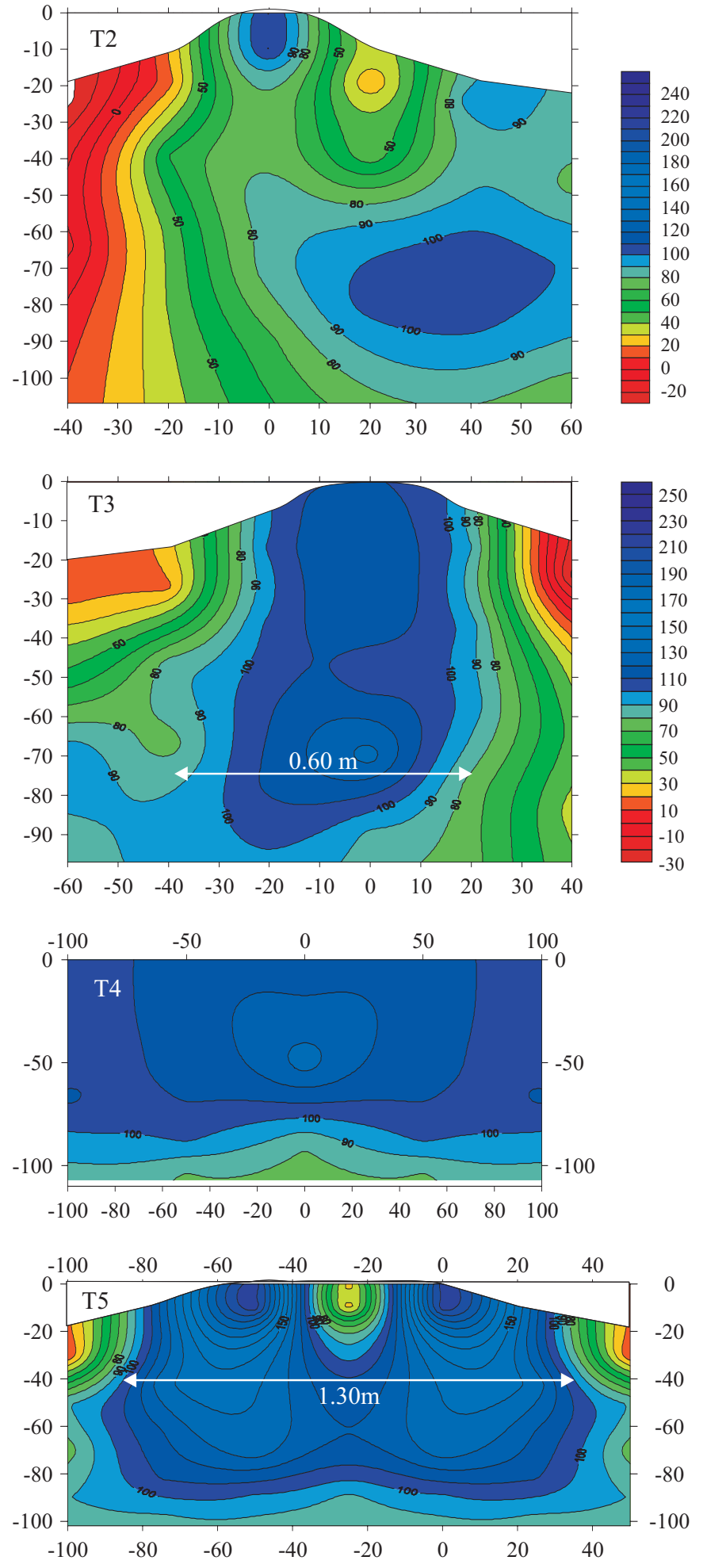

Figura 2. Contenido de agua en el suelo, en los cuatro tratamientos regados

valor 0 indica la posición del gotero y en el caso de la doble línea del T5, los dos goteros están en las posiciones 0 y -60.

El porcentaje de suelo mojado, a 0,30 m de profundidad, calculado respecto a la superficie de la proyección horizontal de la copa fue: T2 16, T3 18, T4 22 y T5 44\%.

En el caso del T4 (microjet), en la Figura 2 se ve que cubre una superficie importante de dos metros de ancho, en la transecta perpendicular a la fila y bajo el emisor. Sin embargo, 
en el sentido de la fila sólo alcanza 1,25 m (datos no mostrados), pues parte del agua es interceptada por la copa.

Es bastante sorprendente también el similar volumen de suelo mojado por el T2 y el T3, a pesar de que la diferencia de caudal de los emisores y la distancia entre ellos era más del doble (1,6 y 4,0 L h-1, 0,40 y 1,00 m, respectivamente).

Estos resultados muestran que en los suelos estratificados y pesados, típicos de la zona frutícola sur del Uruguay, los patrones de mojado fueron diferentes a los esperados de acuerdo a la bibliografía, obteniéndose menores diámetros en todos los casos. Según Keller \& Bliesner (1990) se deberían dar diámetros de mojado superiores a 1,25 m en esta situación.

Por otra parte el incremento de las horas de riego, provocó un aumento del área mojada en los tratamientos con goteros de 2 y $4 \mathrm{~L} \mathrm{~h}^{-1}$ (resultados no presentados). Sin embargo, como consecuencia del aumento del tiempo de riego se incrementaron las pérdidas por percolación, disminuyendo la eficiencia de aplicación del método ya que parte del agua aplicada no fue aprovechada por el cultivo.

Estos resultados concuerdan con los de Pizarro (1990) quien indica un mayor movimiento vertical del agua al aumentar el tiempo de riego afectando muy poco el desarrollo horizontal de la zona mojada.

El único tratamiento que logró aumentar significativamente el porcentaje de suelo mojado fue el T5, que utiliza dos líneas portagoteros.

Los porcentajes de suelo mojado logrados son similares a los recomendados por Keller \& Bliesner (1990). Sin embargo se ha comprobado que en regiones con abundantes lluvias, el porcentaje de mojado debe ser mayor debido a que las raíces se extienden más allá de la zona mojada y la respuesta de las plantas varía directamente con el aumento del área mojada (Zekri \& Parsons, 1988).

\section{Parámetros de crecimiento vegetativo}

Los dos parámetros de crecimiento vegetativo que se presentan en este trabajo tienen un comportamiento similar. En ambos casos la tendencia es que el T3 produce los mayores crecimientos, mientras que el secano (T1) los menores.

Para el peso de poda, los cuatro tratamientos regados no difieren entre sí, pero sí se diferencian estadísticamente del secano $(\mathrm{p}<0,05)$.

En el caso de la sección transversal del tronco, hasta el inicio de la primavera de 2006 el T3 era significativamente mayor que el secano $(\mathrm{p}<0.05)$, mientras que $\mathrm{T} 2$, T4 y T5 no se diferenciaron del T1 ni del T3. Sin embargo y luego de las lluvias ocurridas en octubre, noviembre y diciembre (357 mm en total), el secano tuvo la mayor tasa de crecimiento y en la última medición realizada (10/01/2007) no hubo diferencias entre los tratamientos (Figura 3).

Al comparar el tratamiento con microjet (T4) con los tratamientos de riego por goteo (T2, T3, T5), si bien no se registraron diferencias significativas entre tratamientos, el T3 superó en ambas variables al tratamiento con microjet. Estos resultados son coincidentes con los reportes de Bryla et al. (2003, 2005), quienes obtuvieron un mayor peso de poda y sección transversal del tronco en árboles regados por go-
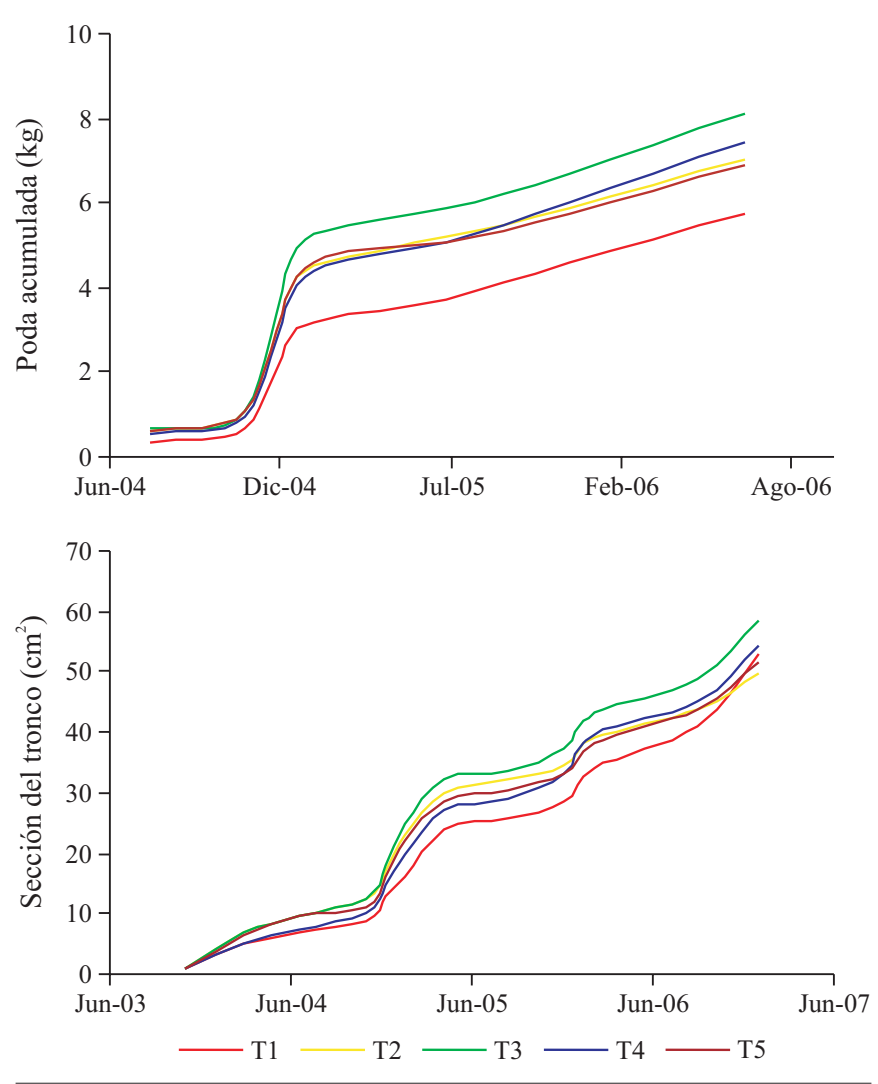

Figura 3. Peso de poda acumulado, en $\mathrm{kg}^{2}$ ábol ${ }^{-1}$ y evolución de la sección transversal del tronco, en $\mathrm{cm}^{2}$, desde el inicio del experimento

teo en comparación con los regados con microjets, atribuyendo estos resultados a la baja eficiencia de aplicación de los microjets.

\section{Parámetros fisiológicos}

El potencial hídrico foliar medido antes del amanecer (entre las 4 y las 6 h), es un indicador del estado hídrico del árbol en equilibrio con el contenido de agua del suelo, ya que se hace en ausencia de transpiración (Garnier \& Berger, 1985).

En las distintas fechas, el secano está siempre con un potencial significativamente más negativo que los tratamientos regados. Entre éstos, si bien existen diferencias significativas, no se dan con un patrón claro. Es así que en la primera fecha el T2 supera al T4, y en la segunda y tercera fecha el T5 supera al T3. En la cuarta fecha no existen diferencias entre los tratamientos regados (Tabla 3).

Es bastante sorprendente verificar que el T3 es generalmente el que tiene el menor potencial de todos los tratamientos regados cuando, de acuerdo a lo visto en el apartado anterior 4 es el que tuvo los mayores crecimientos vegetativos.

De acuerdo a Hsiao et al. (1976), en muchos cultivos el proceso más sensible al estrés hídrico es el crecimiento expansivo. Generalmente, este proceso es aún más sensible al estrés hídrico que la apertura estomática como consecuencia del rol crítico que tiene el turgor en dicho proceso.

Las medidas de potencial hídrico tomadas a media mañana y al mediodía siguen la misma tendencia que las de antes del amanecer. Lo mismo puede decirse del potencial xilemático. 
Las medidas de conductancia muestran una muy buena relación con las de potencial: los tratamientos de menor potencial hídrico tienen también menor conductancia estomática. La excepción a esta norma fue la medida del 10/2/06 a primera hora, en que el secano era el que tenía el menor potencial, pero la menor conductancia la tenía el T5.

Tabla 3. Potencial hídrico foliar (MPa) y conductancia estomática $\left(\mathrm{mMol} \mathrm{cm}^{-2} \mathrm{~s}^{-1}\right)$ en las diferentes fechas y horas del día

\begin{tabular}{|c|c|c|c|c|c|c|}
\hline \multirow[b]{2}{*}{ hora } & \multicolumn{3}{|c|}{ Potencial hídrico } & \multicolumn{3}{|c|}{ Conductancia } \\
\hline & 05:00 & $10: 00$ & 13:00 & 08:00 & $10: 00$ & 13:00 \\
\hline & \multicolumn{3}{|c|}{ 04/01/2005 } & & & \\
\hline $\mathrm{T} 1$ & $-0.82 c$ & $-1.95 c$ & $-1.91 c$ & & & \\
\hline $\mathrm{T} 2$ & $-0.27 \mathrm{a}$ & $-0.99 \mathrm{a}$ & $-1.07 \mathrm{a}$ & & & \\
\hline T3 & $-0.36 a b$ & $-1.36 b$ & $-1.20 a b$ & & & \\
\hline $\mathrm{T} 4$ & $-0.48 b$ & $-1.53 b$ & $-1.50 \mathrm{~b}$ & & & \\
\hline \multirow[t]{2}{*}{$\mathrm{T} 5$} & $-0.39 a b$ & $-1.06 \mathrm{a}$ & $-1.15 \mathrm{a}$ & & & \\
\hline & \multicolumn{3}{|c|}{$13 / 12 / 2005$} & & & \\
\hline $\mathrm{T} 1$ & $-0.62 c$ & $-2.00 \mathrm{~b}$ & $-1.89 b c$ & & & \\
\hline $\mathrm{T} 2$ & $-0.21 a b$ & $-1.55 \mathrm{a}$ & $-1.35 \mathrm{a}$ & & & \\
\hline $\mathrm{T} 3$ & $-0.30 \mathrm{~b}$ & $-1.55 \mathrm{a}$ & $-1.97 \mathrm{c}$ & & & \\
\hline $\mathrm{T} 4$ & $-0.35 a b$ & $-1.46 \mathrm{a}$ & $-1.68 b$ & & & \\
\hline \multirow[t]{2}{*}{$\mathrm{T} 5$} & $-0.19 a$ & $-1.48 \mathrm{a}$ & -1.92 bc & & & \\
\hline & \multicolumn{3}{|c|}{$05 / 01 / 2006$} & \multicolumn{3}{|c|}{$05 / 01 / 2006$} \\
\hline $\mathrm{T} 1$ & $-0.91 c$ & $-2.38 b$ & $-2.60 c$ & $254 \mathrm{~b}$ & $147 \mathrm{~b}$ & $82 \mathrm{c}$ \\
\hline $\mathrm{T} 2$ & $-0.39 a b$ & $-1.93 a b$ & $-1.97 \mathrm{~b}$ & $302 a b$ & $276 \mathrm{a}$ & $203 \mathrm{~b}$ \\
\hline $\mathrm{T} 3$ & $-0.56 \mathrm{~b}$ & $-1.96 a b$ & $-2.03 b$ & $259 \mathrm{~b}$ & $268 a b$ & $236 \mathrm{~b}$ \\
\hline $\mathrm{T} 4$ & $-0.50 a b$ & $-2.17 a b$ & $-2.03 b$ & $386 a$ & 328 a & $272 a b$ \\
\hline \multirow[t]{2}{*}{$\mathrm{T} 5$} & $-0.36 \mathrm{a}$ & $-1.79 \mathrm{a}$ & $-1.70 \mathrm{a}$ & $293 a b$ & $336 \mathrm{a}$ & $320 \mathrm{a}$ \\
\hline & \multicolumn{3}{|c|}{$10 / 02 / 2006$} & \multicolumn{3}{|c|}{$10 / 02 / 2006$} \\
\hline $\mathrm{T} 1$ & $-0.46 \mathrm{~b}$ & & & $1153 \mathrm{a}$ & $594 \mathrm{a}$ & \\
\hline $\mathrm{T} 2$ & $-0.22 \mathrm{a}$ & & & $1177 \mathrm{a}$ & $369 c$ & \\
\hline $\mathrm{T} 3$ & $-0.37 a b$ & & & $1083 a b$ & $544 a b$ & \\
\hline T4 & $-0.36 a b$ & & & $1310 \mathrm{a}$ & $580 a b$ & \\
\hline $\mathrm{T} 5$ & $-0.33 a b$ & & & $810 \mathrm{~b}$ & 408 bc & \\
\hline \multicolumn{4}{|c|}{$16 / 01 / 2007\left(^{\star}\right)$} & \multicolumn{3}{|c|}{$16 / 01 / 2007$} \\
\hline $\mathrm{T} 1$ & & & $-1.54 b$ & & & $175 \mathrm{c}$ \\
\hline $\mathrm{T} 2$ & & & $-1.14 \mathrm{a}$ & & & $403 \mathrm{bc}$ \\
\hline $\mathrm{T} 3$ & & & $-0.93 \mathrm{a}$ & & & $610 \mathrm{ab}$ \\
\hline $\mathrm{T} 4$ & & & $-1.08 \mathrm{a}$ & & & $510 a b c$ \\
\hline $\mathrm{T} 5$ & & & $-1.02 \mathrm{a}$ & & & 746 a \\
\hline \multicolumn{7}{|c|}{$31 / 01 / 2007\left(^{*}\right)$} \\
\hline $\mathrm{T} 1$ & & & $-1.54 c$ & & & \\
\hline $\mathrm{T} 2$ & & & $-1.39 b c$ & & & \\
\hline $\mathrm{T} 3$ & & & $-1.09 \mathrm{a}$ & & & \\
\hline $\mathrm{T} 4$ & & & $-1.21 a b$ & & & \\
\hline $\mathrm{T} 5$ & & & $-1.27 a b c$ & & & \\
\hline
\end{tabular}

Valores seguidos de la misma letra no son diferentes de acuerdo al test de Tukey a $p=0.05$. En las fechas marcadas $\left(^{*}\right)$ se midió el potencial xilemático y no el foliar

\section{Parámetros productivos}

Los parámetros productivos evaluados fueron la evolución del diámetro del fruto, el rendimiento en la cosecha y la calidad de la producción. La primera temporada en que hubo una cosecha comercial fue la 2006/2007, pero en la temporada anterior hubo suficientes frutos para hacer las curvas de crecimiento de los mismos.

\section{Crecimiento de los frutos}

Las curvas de crecimiento de los frutos en ambas tempo- radas (Figura 4) tienen una tendencia similar, con los tratamientos regados por goteo (T2, T3 y T5) con los mayores tamaños, el regado con microjet (T4) tamaño intermedio y el secano (T1) el menor tamaño. Esta tendencia es coincidente con las medidas de agua en el suelo (Figura 1).

En la temporada 2005/2006 las diferencias son mayores hasta enero de 2006 en que, como ya fue comentado anteriormente, comenzaron tres meses de lluvias copiosas. Se nota claramente que la tasa de crecimiento de los frutos en el T4 y T1 es mayor que en el resto (Figura 4).

En la temporada siguiente, por el contrario, los frutos de los tratamientos regados por goteo estaban creciendo a una tasa mayor que los del T4 y T1, hasta que los problemas ya mencionados en el equipo de riego hacen que esa tasa se vuelva menor.

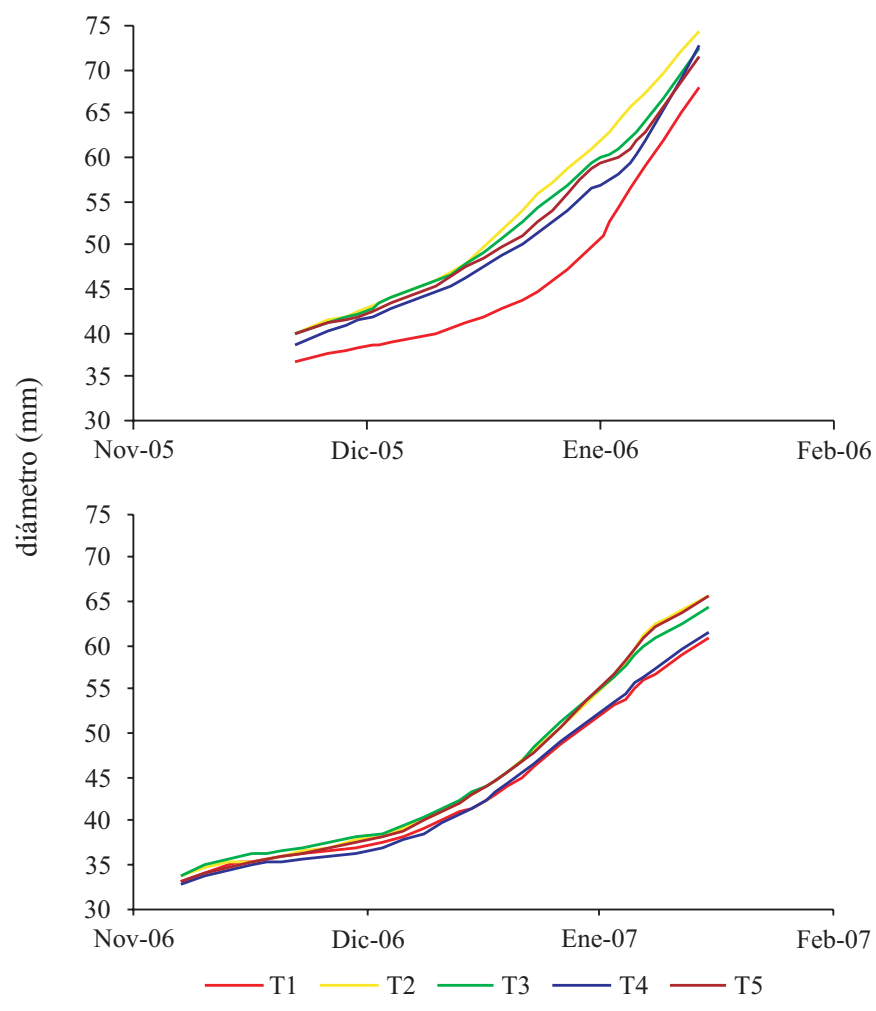

Figura 4. Evolución del diámetro de los frutos en las temporadas 2005/ 2006 y $2006 / 2007$

\section{Producción}

En la primera cosecha del ensayo los cuatro tratamientos regados rindieron significativamente más que el secano (T1), aunque sin diferencias entre ellos (Tabla 4). Sin perjuicio de esta falta de diferencias, se insinúa una tendencia similar a la del crecimiento vegetativo (Figura 3), sobre todo con el peso de poda. Es decir, que el T3 hasta ahora produjo árboles algo más grandes y quizás por eso mismo, con más producción. Se plantea la hipótesis que los efectos de estos tratamientos, en un cultivo perenne, serán acumulativos y se podrán expresar en años sucesivos.

El menor rendimiento de los árboles de secano se debe tanto a un menor número de frutos como a un menor tamaño de los mismos. En otros frutales es conocida la relación 
lineal y negativa entre el número y el tamaño de los frutos (Wiegand \& Swanson, 1982; Castel \& Buj, 1993; GarcíaPetillo, 2002). Por lo tanto, que haya tenido frutos más chicos a pesar de la menor carga, es una clara demostración que ello se debió al estrés hídrico sufrido.

Tabla 4. Resultados de la cosecha 2006/2007

\begin{tabular}{|c|c|c|c|}
\hline & $\begin{array}{l}\text { Rendimiento } \\
\text { (kg árbol }^{-1} \text { ) }\end{array}$ & Número de frutos & $\begin{array}{c}\text { Tamaño } \\
\text { (g fruto-1) }\end{array}$ \\
\hline $\mathrm{T} 1$ & $12.52 \mathrm{~b}$ & $94 \mathrm{c}$ & 134 b \\
\hline $\mathrm{T} 2$ & $19.87 \mathrm{a}$ & $132 \mathrm{ab}$ & 152 \\
\hline T3 & $22.11 \mathrm{a}$ & $145 \mathrm{a}$ & 157 a \\
\hline $\mathrm{T} 4$ & 19.91 a & $130 \mathrm{ab}$ & $155 a$ \\
\hline $\mathrm{T} 5$ & $17.50 \mathrm{a}$ & 110 bc & $166 a$ \\
\hline
\end{tabular}

El T5 fue, de los tratamientos regados, el que tuvo el menor número de frutos, pero, probablemente debido a ello, los de mayor tamaño promedio aunque sin diferir significativamente de los otros. Se puede especular que este tratamiento tuvo menor cantidad de frutos por ser los árboles de menor tamaño entre los regados (Figura 3).

\section{Distribución por calibres}

Para los fruticultores es de importancia no sólo obtener altas producciones, sino que éstas sean de los calibres de mayor valor comercial (especial y grande). La Figura 5 muestra la distribución por calibres comerciales de la pri-
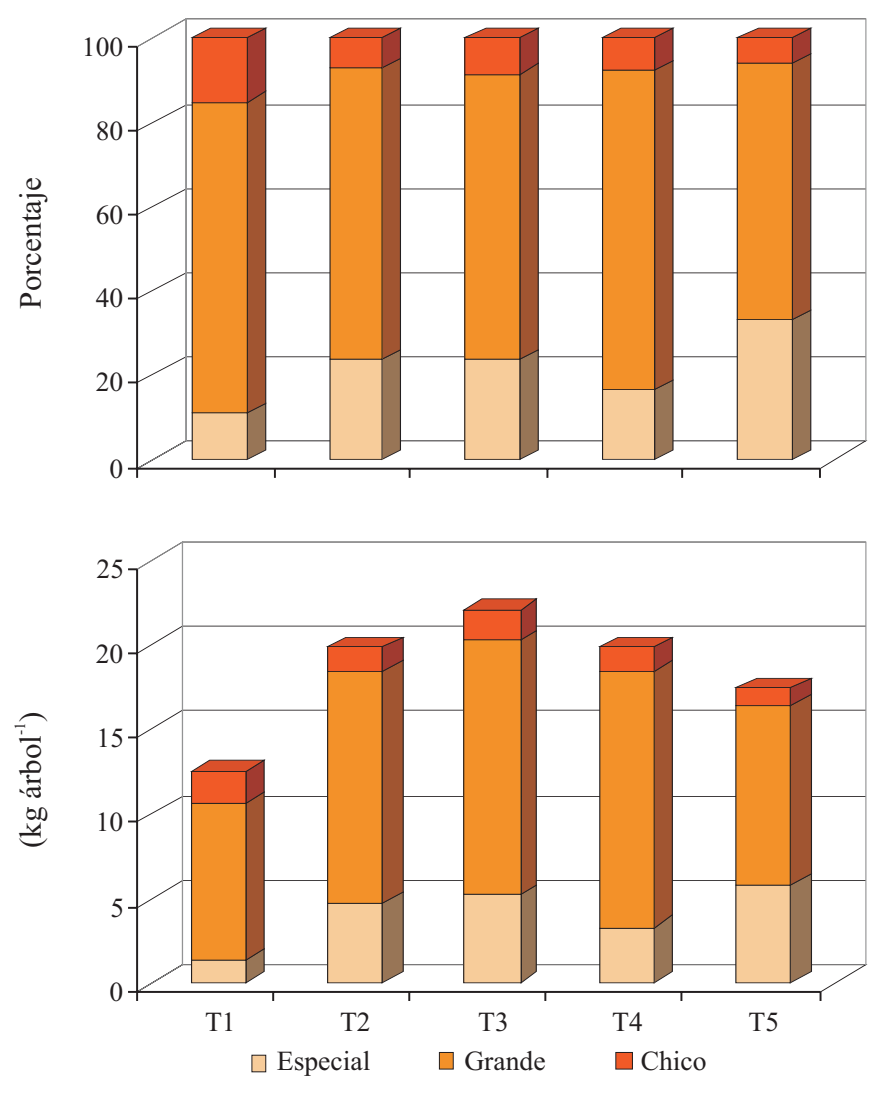

Figura 5. Distribución por calibres (en porcentaje y en $\mathrm{kg}$ árbol ${ }^{-1}$ ) de la producción del ensayo en la temporada 2006/2007 mera cosecha, expresada tanto en porcentaje como en producción de cada calibre.

Los tratamientos regados por goteo (T2, T3 y T5) tienen una mayor producción de los frutos del calibre "Especial” (de más de 180 g) que son los de mayor valor comercial. El secano (T1) es el de menor producción de estos frutos, mientras que el tratamiento regado por microjet (T4) tiene una producción intermedia.

Estadísticamente (análisis no presentado), el T4 no se diferencia del T2 y T3, ni tampoco del T1. Los frutos del calibre "Chico" son siempre los de menor valor comercial, y en situaciones de oferta abundante de duraznos, pueden ser descartes sin valor comercial. El T1 produjo alrededor de un $15 \%$ de estos frutos de descarte, el doble aproximadamente que el promedio de los cuatro tratamientos regados.

\section{CONCLUSIONES}

1. En las variables peso de poda, potencial hídrico foliar y xilemático, conductancia estomática, crecimiento de frutos, rendimiento y tamaño promedio de los frutos, los tratamientos que recibieron riego no se diferenciaron estadísticamente entre sí, pero todos presentaron valores significativamente superiores al secano $(\mathrm{p}<0.05)$.

2. El porcentaje de volumen de suelo mojado regando con una sola línea portagoteros por fila de árboles (T2 y T3) fue similar (16 y 18\%), independiente de los distintos caudales y distancias entre ellos (1,6 y 4,0 L h-1, 0,40 y 1,00 m, respectivamente).

3. Usando microjets no se logró aumentar significativamente el porcentaje del volumen de suelo mojado, llegando sólo a $22 \%$ de la proyección de la copa, debido a las condiciones ya discutidas en el texto.

4. Regando con dos líneas portagoteros por fila sí se dio un aumento significativo del porcentaje de volumen de suelo mojado (44\%).

5. No se evidenció ninguna respuesta entre los tratamientos regados al porcentaje de suelo mojado para todas las variables analizadas. En ningún caso el T5, que mojaba más del doble de volumen que los otros tratamientos, tuvo una respuesta diferencial.

\section{LITERATURA CITADA}

Allen, R. G.; Pereira, L. S.; Raes, D.; Smith, M. Crop evapotranspiration. Guideline for computing crop water requirements. FAO Irrigation and Drainage Paper n 56, Rome: FAO, 1998. 300p.

Black, J. D. F.; West, D. W. Water uptake by an apple tree with various proportions of the root system suply with water. In: International Drip Irrigation Congress, 2, 1974, Riverside. Proceedings ... Riverside: California University, 1974. p.432-433.

Bryla, D. R.; Dickson, E.; Shenk, R.; Johnson, R. S.; Crisosto, C. H.; Trout, T. J. Influence of irrigation method and sheduling on patterns of soil and tree water status and Its relation to yield and fruit quality in peach. HortScience, v.40, n.7, p.2118-2124, 2005.

R. Bras. Eng. Agríc. Ambiental, v.14, n.1, p.17-24, 2010. 
Bryla, D. R.; Trout, T. J.; Ayars J. E.; Johnson, R. S. Growth and production of young peach trees irrigated by furrow, microjet, surface drip, or subsurface drip systems. HortScience, v.38, n.7, p.1112-1116, 2003.

Castel, J. R.; Buj, A. Riego por goteo deficitario en naranjos adultos "Salustiana” durante siete años. Revista Investigación Agraria: Producción y Protección Vegetal, v.8, n.2, p.191-204, 1993.

Durán, A.; Califra, A.; Molfino, J. H.; Lynn W. Keys to soil taxonomy for Uruguay. Washington: United States Department of Agriculture, Natural Resources Conservation Service, 2005. 77p.

Fereres, E. Papel de la fisiología vegetal en la microirrigación. Recomendaciones para el manejo mejorado. In: Seminario Latinoamericano de Microirrigación, 4, 1981, Barquisimeto. Memorias ... Barquisimeto: IICA, 1981. p.23.

García-Petillo, M. Respuesta a diferentes manejos del riego y balance hídrico de un huerto de cítricos. Valencia: Universidad Politécnica de Valencia, Escuela Técnica Superior de Ingenieros Agrónomos. 2002. 194p. Tesis Doctoral

García-Petillo, M.; Hayashi, R.; Puppo, L.; Morales, P. Desarrollo del bulbo húmedo bajo riego localizado en suelos estratificados del Uruguay. En: Congreso Internacional de Riego y Drenaje Cuba-Riego, 2 , 2005, La Habana. Memorias... La Habana: Instituto de Investigación de Riego y Drenaje, 2005. CD Rom.

García-Petillo, M.; Puppo, L.; Chamorro, A.; Hayashi, R. Effects of drip irrigation water amount and soil wetted volume on "Washington Navel” orange yield. Acta Horticulturae, v.646, p.101-106, 2004.

Garnier, E.; Berger, A. Testing water potencial in peach trees as indicator of water stress. Journal of Horticultural Science, v.60, n.1, p.47-56, 1985.

Gispert, J. R. Evaluación del volumen de suelo húmedo en microirrigación. Influencia del porcentaje de este volumen sobre el comportamiento del olivo (Olea Europea L. cult. "Arbequina”). Estudios de la Zona no Saturada del Suelo, v.6, p.51-57, 2003.

Hsiao, T. C.; Fereres, E.; Acevedo, E.; Henderson, D. W. Water stress and dynamics of growth and yield of crop plants. In: Lange, O. L., Kappen, L., Schulze, E. D. (ed), Ecological studies. Analysis and synthesis, v.19. Water and plant life. Berlin: Springer-Verlag. 1976. p.281-305.
Keller, J. Trickle irrigation. Section 15-7. National engineering handbook. Glendora: Soil Conservation Service, 1978. 129p.

Keller, J.; Bliesner, R. Sprinkle and trickle irrigation. New York: Van Nostrand Reinhold, 1990. 652p.

Koo, R. C. J.; Smajstrla, A. G. Trickle irrigation of citrus on sandy soils in a humid region. In: International Drip Irrigation Congress, 3, 1985, Fresno. Proceedings... Fresno: ASAE, 1985. p.212-219.

López, J. R.; Hernández, J. M.; Pérez, A.; González, J. F. Riego localizado. Madrid: Ediciones Mundi-Prensa, 1992. 405p.

MGAP - Ministerio de Ganadería Agricultura y Pesca. Encuesta frutícola DIEA http://www.mgap.gub.uy/Diea/Encuestas/ Se240/SE240_FRUTICOLA_06.htm. 30 Nov. 2006.

Moreshet, S.; Cohen, Y.; Fuchs, M. Response of mature "Shamouti" orange trees to irrigation of different soil volumes at similar levels of available water. Irrigation Science, v.3, p.223-236, 1983.

Pizarro, F. Riegos localizados de alta frecuencia (RLAF) goteo, microaspersión, exudación. Madrid: Ediciones Mundi-Prensa, 1990. 471p.

Scholander, P. F.; Hammel, H. T.; Bradstreet, E. D.; Hemmingsen, E. A. Sap pressure in vascular plants. Science, v.148, p.339-346, 1965.

Shackel, K. The use of midday stem water potential for irrigation scheduling and water stress diagnosis in prune and almond. International Symposium Irrigation and Water Relations in Grapevines and Fruit Trees, 2001, Mendoza. Proceedings... Mendoza: ISHS. 2001. CD. Rom.

Wiegand, C. L.; Swanson, W. A. Citrus response to irrigation: II. Fruit yield, size and number. Journal Rio Grande Valley Horticultural Society, v.35, p.87-95, 1982.

Willoughby, P.; Cockroft, B. Changes in root patterns of peach trees under trickle irrigation. In: International Drip Irrigation Congress, 2, 1974, Riverside. Proceedings... Riverside: University of California, 1974. p.439-442.

Zekri, M.; Parsons, L. R. Water relations of grapefruit tree in response to drip, microsprinkler irrigation. Journal of American Society of Horticultural Science, v.113, n.6, p.819-823, 1988. 